\title{
Are SPECT measurements of myocardial blood flow and flow reserve ready for clinical use?
}

\author{
Ernest V. Garcia
}

Published online: 14 October 2014

(C) Springer-Verlag Berlin Heidelberg 2014

The well-documented diagnostic accuracy of SPECT myocardial perfusion imaging (MPI) for detecting coronary artery disease (CAD) has promoted its widespread clinical use. Nevertheless, the success of SPECT MPI has been attained using the basic SPECT camera design which is over 50 years old [1], using the basic filtered backprojection reconstruction algorithm which is even older, dating to over 90 years ago [2], and using ${ }^{99 \mathrm{~m}} \mathrm{Tc}$-based perfusion agents with limited extraction fractions [3]. Moreover, although SPECT MPI is inherently a digital quantitative technique, our clinical approach to quantifying hypoperfusion has depended on a database approach where a patient's left ventricular perfusion pattern is statistically compared to an expected normal perfusion pattern generated from patients with a low likelihood of CAD [4-6]. In spite of the success of SPECT MPI using these established conventional approaches, recent innovations are poised to bring SPECT MPI to the next level. In a recent article in EJNMMI, Hsu et al. [7] report on the use of dynamic SPECT with a SPECT/CT camera to measure absolute myocardial blood flow (MBF) and MBF reserve (MBFR) in patients. The authors conclude that their flow quantitation method is a clinically effective approach to enhancing CAD detection. Thus it is pertinent to ask: are SPECT measurements of MBF and MBFR ready for clinical use?

Perhaps in a patient-centered environment the first pertinent question should be: how will these measurements help our patients? It has been posited that measurements of MBF and MBFR should not be limited to the role of gatekeeper to the catheterization laboratory but should be used in the more important role of gatekeeper to revascularization [8]. So this approach should not only limit the referral of the $60 \%$ of

E. V. Garcia $(\bowtie)$

Department of Radiology and Imaging Sciences, Emory University,

101 Woodruff Circle, Room 1203, Atlanta 30322, GA, USA

e-mail: ernest.garcia@emory.edu patients who are found not to have obstructive disease in the catheterization laboratory [9] but should also, in the $40 \%$ with obstructive disease, guide the interventionist as to which vessels are truly flow-limiting and have the potential for successful revascularization. As such, the measurements fulfill the mantra of today, i.e., an imaging test should not only yield a correct diagnosis but should also guide successful therapy and thus be directly associated with a patient's outcome that can be used as evidence of the value of the test.

The next pertinent question should be: with today's SPECT instrumentation, radiopharmaceuticals and quantification software, are the measurements of absolute MBF and MBFR accurate and reproducible enough for clinical use? Limited by the lack of dynamic SPECT, early investigations of MBFR measurements with SPECT used first-pass dynamic planar imaging of tetrofosmin [10] and sestamibi [11] to record the input function followed by SPECT without attenuation correction for myocardial sampling. A simple microsphere model was used to measure MBF and/or MBFR. Dynamic SPECT and compartmental modeling were also used to investigate imaging with teboroxime [12] albeit, at the time, there were very few SPECT cameras that could perform this fast dynamic acquisition. These investigations had in common that the methodology was applied to small patient populations or in animal experiments. All of these studies showed the feasibility of measuring MBF and particularly MBFR with SPECT.

In recent years manufacturers have begun to break away from the conventional SPECT imaging approach to create innovative designs for dedicated cardiac imagers. The designs of these imagers have in common that all available detectors are constrained to imaging just the cardiac field of view. These new designs vary in the number and type of scanning or stationary detectors, and whether $\mathrm{NaI}$ or solid-state detectors are used $[13,14]$, but have in common an increase in count sensitivity over conventional SPECT up to a factor of 10 [15]. Similarly, iterative reconstruction has significantly evolved 
allowing the physical correction of scatter, attenuation, resolution changes with depth, and image noise. Although these might sound familiar, today's techniques are more accurate than those used even 10 years ago. Now instead of implementing simple assumptions as in the past, the entire imaging process is modeled to better correct for these physical phenomena. Two groups have reported on the feasibility of using two of these new heart-centered CZT SPECT detector systems to measure MBF [16] and MBFR [16, 17].

Hsu et al. [7], in this issue of EJNMMI, report on the use of dynamic SPECT with a SPECT/CT camera and iterative reconstruction with comprehensive correction methodology to measure absolute MBF and MBFR in humans. These investigators chose to use a standard dual-detector camera equipped with parallel-hole collimators. Somewhat less conventional was the fact that the camera was able to perform a $180^{\circ}$ arc acquisition, back and forth, every $10 \mathrm{~s}$ to record the input function as the first pass of the sestamibi tracer through the ventricles. Also not typical was their use of iterative reconstruction with comprehensive correction methodology which corrected for scatter, attenuation, resolution changes with depth, and image noise. The approach of these investigators was inspired by the success of PET measurements of MBF and MBFR but motivated by the need to provide flow measurements in situations in which PET and solid-state SPECT are not available. They also chose to use the FlowQuant program developed at the University of Ottawa validated for measuring flow with ${ }^{82} \mathrm{Rb}$ [18] but modified to use a single compartment model and the sestamibi extraction fraction.

To determine if a diagnostic method is ready for clinical use, the simple but pertinent question is whether, for a given clinical application in a given patient, the error of the measurement is such that we can reasonably separate, in this case, normal from abnormal MBF and/or MBFR. Hsu et al. [7] report that there was a statistically significant difference in stress MBF and MBFR between their 13 patients with CAD and 8 patients with no significant lesions $(p=.02$ and $p<.001$, respectively). Even though the patient population was quite small these results are consistent in proving the feasibility of the technique. Yet comparing the flow results on a patient by patient basis there was a clinically significant overlap between the CAD and non-CAD patients even though the $13 \mathrm{CAD}$ patients had an unusually high frequency of multivessel disease $(62 \%)$. In their population, the AUC for MBFR from ROC analysis was significantly greater than the AUCs for the visual summed stress score and summed difference score, but stress MBF was not.

The more convoluted answer as to whether SPECT measurements of MBF and MBFR are ready for prime time is whether both efficacy and effectiveness have been established keeping in mind that we should try to avoid the mistakes of the past when introducing new technology. Because all of the studies on the measurement of MBF and MBFR with SPECT have dealt with very small patient populations or have involved animal experiments, there is no evidence of the efficacy and particularly effectiveness of these measurements in the clinical environment. What is clearly established to date with SPECT is the feasibility of performing these myocardial flow measurements. Feasibility is quite important and necessary for the field to understand that there is no theoretically inherent limitation to the SPECT approach for measuring MBF. Yet feasibility alone is not enough to embark on clinical use.

This brings to light the mistakes of the past when introducing new technology. Two technologies come to mind: firstpass radionuclide angiography and SPECT attenuation correction. Even though most experts would agree that both of these techniques have been shown to be highly efficacious, the frequency of their current use in patient studies is disappointing. The limiting factor in the use of first-pass studies was the complaint that it was too difficult to perform, in part because of the need for a bolus injection, synchronized start of the acquisition, fast counting cameras, fast framing rates, and so on. The initial complaint about SPECT attenuation correction was that it did not work, i.e., that it reduced the diagnostic yield. Later when the methodology matured and was shown to work in daily routine, the complaint was that there was no financial incentive to perform this additional task. The introduction of these technologies shared the common mistake of rushing to clinical use before they were ready. Readiness factors include the commercial availability of the right equipment, right radiopharmaceutical, easy to use and robust software, and perhaps most importantly appropriate training for the users.

As we learned with SPECT attenuation correction, once a new technology develops a bad reputation it takes years to create the trust for clinical use, even after all the technical problems have been fixed. Moreover, had these techniques obtained wide acceptance, MBF and MBFR could be more readily measured with SPECT today. These SPECT flow measurements require first-pass techniques to capture the input function, SPECT attenuation (and other physical phenomena) correction for measuring absolute concentration, heart-centered, multidetector, high count sensitivity cameras to reduce noise, SPECT tracers with higher extraction fraction (such as teboroxime [12] and ${ }^{123}$ I-rotenone [19]) and flow quantification software similar to that used in PET as applied by Hsu et al. [7].

Importantly, although comprehensive correction methodology considerably improves the accuracy of an absolute measurement of concentration, it tends to increase the error with which the measurement is made. This is particularly true for conventional SPECT cameras with low count density in the dynamic acquisitions and a clear disadvantage as compared to PET flow measurements. This is further compounded in SPECT with the use of tracers with a low myocardial extraction fraction such as sestamibi and tetrofosmin. 
Although it is known how to correct for these limited extraction fractions, the correction in the presence of image noise further propagates the noise and thus the ability to differentiate normal from abnormal flows in a specific patient.

Perhaps one answer would be to use higher radiopharmaceutical doses. Indeed Hsu et al. used conventional protocol doses of $370 \mathrm{MBq} / 1,000 \mathrm{MBq}$ for the stress/rest sestamibi studies, which result in approximately $12 \mathrm{mSv}$ exposure to the patient. Because of concerns about the risk to patients from radiation, the trend is moving in the opposite direction, i.e., towards significant dose reductions. The American Society of Nuclear Cardiology published an information statement [20] recommending that laboratories use imaging protocols that achieve on average a radiation exposure of less than or equal to $9 \mathrm{mSv}$ in $50 \%$ of studies. Although there are many different protocols that may be implemented to accomplish this exposure goal, use of the more efficient hardware/software described above would greatly facilitate the achievement of this goal and allow improved SPECT measurements of myocardial flow compared with conventional cameras.

Today SPECT cardiovascular imaging is a fast-changing field in which made major leaps in instrumentation hardware and reconstruction software have led to imaging performance consistent with the potential to accurately measure MBF and MBFR. Sestamibi and tetrofosmin radiopharmaceuticals suitable for blood flow measurements, albeit it with limited myocardial extraction fractions, are commercially available. Chemists know how to develop and have developed and validated SPECT tracers with higher extraction fractions than that of ${ }^{82} \mathrm{Rb}$, clearly showing that there is no inherent limitation of SPECT radiopharmaceuticals for measuring myocardial flow. Hsu et al. [7] have shown how the validated software used for measuring MBF with PET agents can be adapted to model SPECT tracers. Finally, although there are no real inherent limitations to the measurement of MBF and MBFR with SPECT and investigations like that of Hsu et al. [7] have shown this feasibility, the efficacy and effectiveness [8] of these SPECT measurements must be established before the method is ready for clinical use.

\section{References}

1. Anger HO. Scintillation camera. Rev Sci Instrum. 1958;29(1):27-33.

2. Radon J. Uber due bestimmung von funktionen durch ihre intergralwerte langsgewisser mannigfaltigkeiten (on the determination of functions from their integrals along certain manifolds). Ber Saechsische Akad der Wiss. 1917;29:262-77.

3. Beller GA, Bergmann SR. Myocardial perfusion imaging agents: SPECT and PET. J Nucl Cardiol. 2004;11:71-86.
4. Ficaro EP, Lee BC, Kritzman JN, Corbett JR. Corridor4DM: the Michigan method for quantitative nuclear cardiology. J Nucl Cardiol. 2007; 14:455-65.

5. Germano G, Kavanagh PB, Slomka PJ, Van Kriekinge SD, Pollard G, Berman DS. Quantitation in gated perfusion SPECT imaging: the Cedars-Sinai approach. J Nucl Cardiol. 2007;14:433-54.

6. Garcia EV, Faber TL, Cooke CD, Folks RD, Chen J, Santana C. The increasing role of quantification in nuclear cardiology: the Emory approach. J Nucl Cardiol. 2007;14:420-32.

7. Hsu B, Chen FC, Wu TC, Huang WS, Hou PN, Chen CC, Hung GU. Quantitation of myocardial blood flow and myocardial flow reserve with $99 \mathrm{mTc}$-sestamibi dynamic SPECT/CT to enhance detection of coronary artery disease. Eur J Nucl Med Mol Imaging. 2014. doi:10. 1007/s00259-014-2881-9

8. Garcia EV. Are absolute myocardial blood flow PET measurements ready for clinical use? J Nucl Cardiol. 2014;21:857-8.

9. Patel MR, Peterson ED, Dai D, Brennan JM, Redberg RF, Anderson $\mathrm{HV}$, et al. Low diagnostic yield of elective coronary angiography. $\mathrm{N}$ Engl J Med. 2010;362:886-95.

10. Sugihara H, Yonekura Y, Kataoka K, Fukai D, Kitamura N, Taniguchi Y. Estimation of coronary flow reserve with the use of dynamic planar and SPECT images of Tc-99m tetrofosmin. J Nucl Cardiol. 2001;8:575-9.

11. Storto G, Cirillo P, Vicario ML, Pellegrino T, Sorrentino AR, Petretta $\mathrm{M}$, et al. Estimation of coronary flow reserve by Tc- $99 \mathrm{~m}$ sestamibi imaging in patients with coronary artery disease: comparison with the results of intracoronary Doppler technique. J Nucl Cardiol. 2004;11(6): $651-5$.

12. Di Bella EV, Ross SG, Kadrmas DJ, Khare HS, Christian PE, McJames S, et al. Compartmental modeling of technetium- $99 \mathrm{~m}$ labeled teboroxime with dynamic single photon emission computed tomography: comparison with static thallium-201 in a canine model. Invest Radiol. 2001;36:178-85.

13. Garcia EV, Faber TL, Esteves FP. Cardiac dedicated ultrafast SPECT cameras: new designs and clinical implications. J Nucl Med. 2011;52:210-7.

14. Sharir T, Slomka PJ, Berman DS. Solid-state SPECT technology: fast and furious. J Nucl Cardiol. 2010;17:890-6.

15. Imbert L, Poussier S, Franken PR, Songy B, Verger A, Morel O, et al. Compared performance of high-sensitivity cameras dedicated to myocardial perfusion SPECT: a comprehensive analysis of phantom and human images. J Nucl Med. 2012;53:1897-903.

16. Pazhenkottil AP, Nkoulou R, Kuest S, Ghadri J, Fiechter M, Fuchs T, et al. Absolute coronary blood flow and coronary flow reserve assessed by gated SPECT with cadmium-zinc-telluride detectors: a direct comparison with 13N-ammonia PET. J Am Coll Cardiol. 2013;61(10):E1005 (abstract). doi:10.1016/S0735-1097(13)61005-0

17. Ben-Haim S, Murthy VL, Breault C, Allie R, Sitek A, Roth N, et al. Quantification of myocardial perfusion reserve using dynamic SPECT imaging in humans: a feasibility study. J Nucl Med. 2013;54:873-9.

18. Lortie M, Beanlands RS, Yoshinaga K, Klein R, Dasilva JN, DeKemp RA. Quantification of myocardial blood flow with $82 \mathrm{Rb}$ dynamic PET imaging. Eur J Nucl Med Mol Imaging. 2007;34: $1765-74$.

19. Marshall RC, Powers-Risius P, Reutter BW, Taylor SE, VanBrocklin HF, Huesman RH, et al. Kinetic analysis of 125I-iodorotenone as a deposited myocardial flow tracer: comparison with $99 \mathrm{mTc}$-sestamibi. J Nucl Med. 2001;42(2):282-4.

20. Cerqueira MD, Allman KC, Ficaro EP, Hansen CL, Nichols KJ, Thompson $\mathrm{RC}$, et al. Recommendations for reducing radiation exposure in myocardial perfusion imaging. J Nucl Cardiol. 2010;17:709-18. 